\title{
Insertional Mutagenesis
}

National Cancer Institute

\section{Source}

National Cancer Institute. Insertional Mutagenesis. NCI Thesaurus. Code C17377.

Mutagenesis of DNA by the insertion of one or more bases. 